Supporting Information for:

\title{
Responsive Photonic Crystal Microcapsules of Block Copolymers with Enhanced Monochromaticity
}

Yi Yang, Yu Chen, Zaiyan Hou, Fan Li, Mengjun Xu, Yuanyuan Liu, Di Tian, Lianbin Zhang, Jiangping $\mathrm{Xu}$ * and Jintao Zhu*

State Key Lab of Materials Processing and Die \& Mould Technology and Key Lab of Materials Chemistry for Energy Conversion \& Storage (HUST) of Ministry of Education, School of Chemistry and Chemical Engineering, Huazhong University of Science and Technology (HUST), Wuhan 430074, China

E-mail: jiangpingxu@hust.edu.cn (J. X.)

jtzhu@mail.hust.edu.cn (J. Z.) 


\section{Characterization Section}

Optical characterization: The formation of PC microcapsules was monitored by Olympus IX71 inverted optical microscope under transmission mode. The structural colors exhibited by the PC microcapsules were observed under reflection mode. The photographs were captured by a high-speed CCD connected to the microscope. The reflection spectra of the microspheres were measured by using a fiber-optic spectrometer (USB4000, Ocean Optics Inc.) connected to the microscope.

Cross-sectional characterization of the microcapsules: $5 \mathrm{~mL}$ of PC microspheres suspension was concentrated to $0.5 \mathrm{~mL}$ to remove PVA and then freeze-dried in a lyophilizer. The PC microcapsules were then embedded in epoxy resin and cut by a diamond knife (Electron Microscopy Sciences) into ultra-thin cross-sections (thickness: $100 \mathrm{~nm}$ ) using a Leica ultra-microtome (UCT-GA-D/E-1/00). Transmission Electron Microscope (TEM) samples were prepared by transferring the cross-sections on 300 mesh copper grids and then stained with iodine vapors for $1 \mathrm{~h}$ to enhance the contrast between P2VP and PS domains. TEM investigation was performed on a JEOL 2100 facility at an acceleration voltage of $200 \mathrm{kV}$.

Confocal laser scanning microscopy (CLSM) measurements: Hydrophobic fluoresce dye, Nile red, was added into chloroform before emulsification and finally located in the shell of PC microcapsules. After removing residual dyes absorbed on the surface, the PC microcapsules were put on a glass substrate for measurement. All fluorescence images were captured with an Olympus biological CLSM (FV1200) and a 564 nm laser was used for the excitation of Nile red. Fluorescence distribution was checked by $\mathrm{Z}$ axis scanning from top to bottom of the PC microcapsules. The interval of each scan was $5 \mu \mathrm{m}$. 
UV-Vis absorption spectrum: The supernatant containing released R6G was added into a $100 \mu \mathrm{L}$ cuvette for measurement at $25{ }^{\circ} \mathrm{C}$. The measuring range of the absorption spectrum ranged from 400 $\mathrm{nm}$ to $1100 \mathrm{~nm}$ and deionized water was used as reference. 


\section{Supporting Figures:}
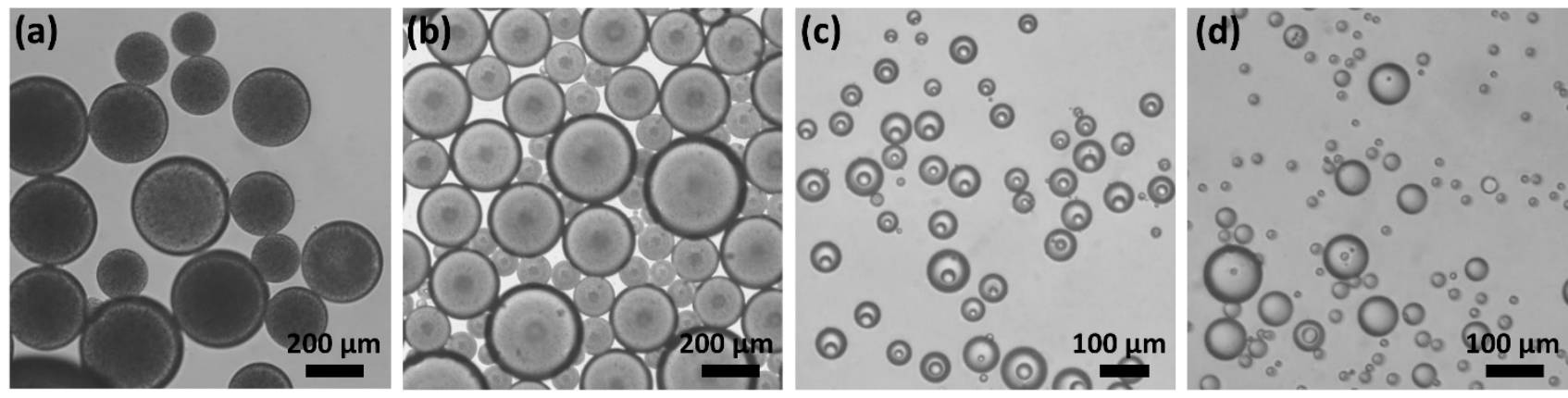

Figure S1. (a-d) OM images of the emulsion droplets after evaporating for 2 h, 4 h, 10 h, and 16 h, respectively. The height of aqueous phase was kept at $10 \mathrm{~mm}$. The concentration of PS- $b$-P2VP was $5 \mathrm{mg} / \mathrm{mL}$ and the concentration of AOT was $10 \mathrm{mg} / \mathrm{mL}$ in all cases. 

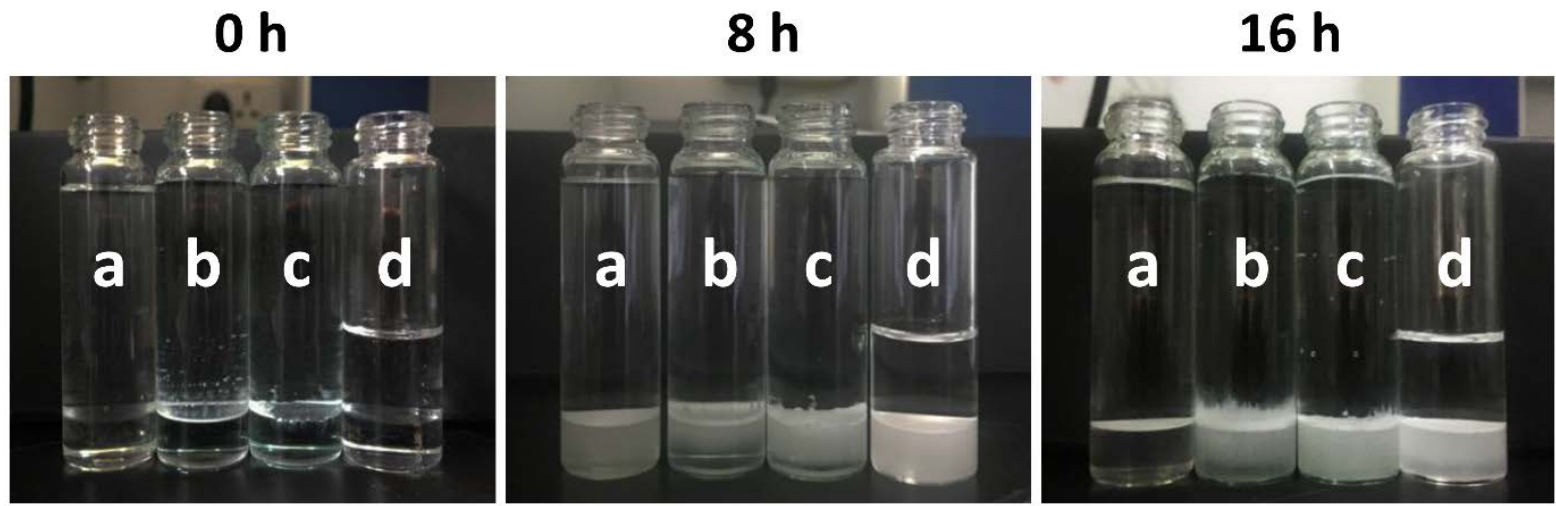

Figure S2. Photographs showing the self-emulsification phenomena of four chloroform/water system. The photos were taken at $0 \mathrm{~h}, 8 \mathrm{~h}$, and $16 \mathrm{~h}$ after adding water (top layer in the vial) to chloroform (bottom layer in the vial). The liquids in the vials were (a) water and $10 \mathrm{mg} / \mathrm{mL}$ AOT chloroform solution, (b) water and $30 \mathrm{mg} / \mathrm{mL}$ AOT chloroform solution, (c) $1.5 \mathrm{mg} / \mathrm{mL}$ AOT water solution and $10 \mathrm{mg} / \mathrm{mL}$ AOT chloroform solution, (d) water and $10 \mathrm{mg} / \mathrm{mL}$ AOT chloroform solution, respectively. The volume ratios of chloroform to water were 1:6 in (a-c), while 1:2 in (d).

As shown in Figure S1a-d, when the concentration of AOT was relatively low $(10 \mathrm{mg} / \mathrm{mL})$, water droplets would escape from the emulsion droplets when the evaporation of chloroform lasted for over $16 \mathrm{~h}$, indicating the decreasing stability of water droplets during evaporation. We ascribed the escape of inner water droplets from the double emulsion to the diffusion of water-soluble AOT from oil phase to the external water phase, due to the concentration gradient of AOT between oil phase and water phase. Therefore, we proposed three methods, including 1) increasing the initial concentration of AOT in chloroform (over $30 \mathrm{mg} / \mathrm{mL}$ ); 2) introducing additional AOT to the outer aqueous media $(1.5 \mathrm{mg} / \mathrm{mL}) ; 3)$ increasing the volume ratio of chloroform to aqueous phase, to sustain the AOT concentration in oil phase or slow down the diffusion of AOT during evaporation.

The experiments shown in Figure S2 aimed at verifying whether the proposed methods are effective in maintaining the stability of water droplets in chloroform. In experiment a, the AOT 
chloroform solution became turbid and turned transparent in $16 \mathrm{~h}$. This phenomenon indicated that self-emulsification occurred at early stage, but the emulsions were not stable over time. In experiment b-d, self-emulsification phenomenon could still be observed when the samples were stored for over $16 \mathrm{~h}$, indicating the water droplets were more stable in these cases. In experiment b, increasing AOT concentration in chloroform enabled sufficient AOT to stabilize the inner water droplets of the double emulsions for a long time. In experiment c, introducing additional AOT to the outer aqueous phase decreased the concentration contrast of AOT between oil phase and water phase, resulting in the slowing down of AOT diffusion. Therefore, there were enough AOT stabilizing the inner water droplets in the double emulsions in experiment b and c. In experiment d, decreasing the volume fraction of water phase resulted in faster raise of AOT concentration in water phase during diffusion, which could also relieve the transfer of AOT to the water phase. As a result, the three methods could effectively retard the escape of inner water droplets from the double emulsion. 


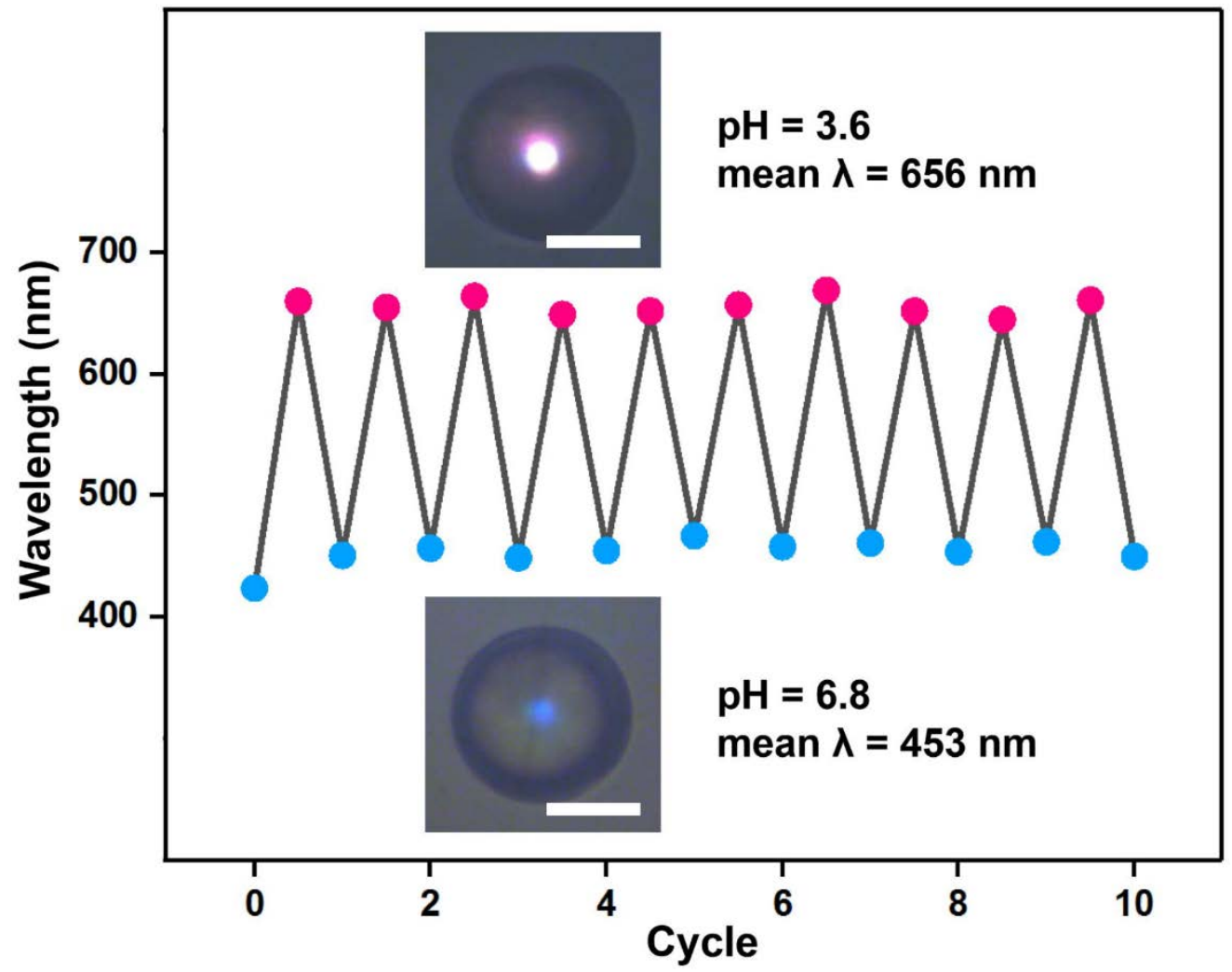

Figure S3. Plot shows the reversible color change of the $\mathrm{PS}_{213 \mathrm{k}}-b-\mathrm{P} 2 \mathrm{VP} 215 \mathrm{k}$ microcapsules immersed in aqueous solutions by switching $\mathrm{pH}$ value between 3.6 and 6.8. The experiments were performed for 10 cycles. The scale bars are $50 \mu \mathrm{m}$. 


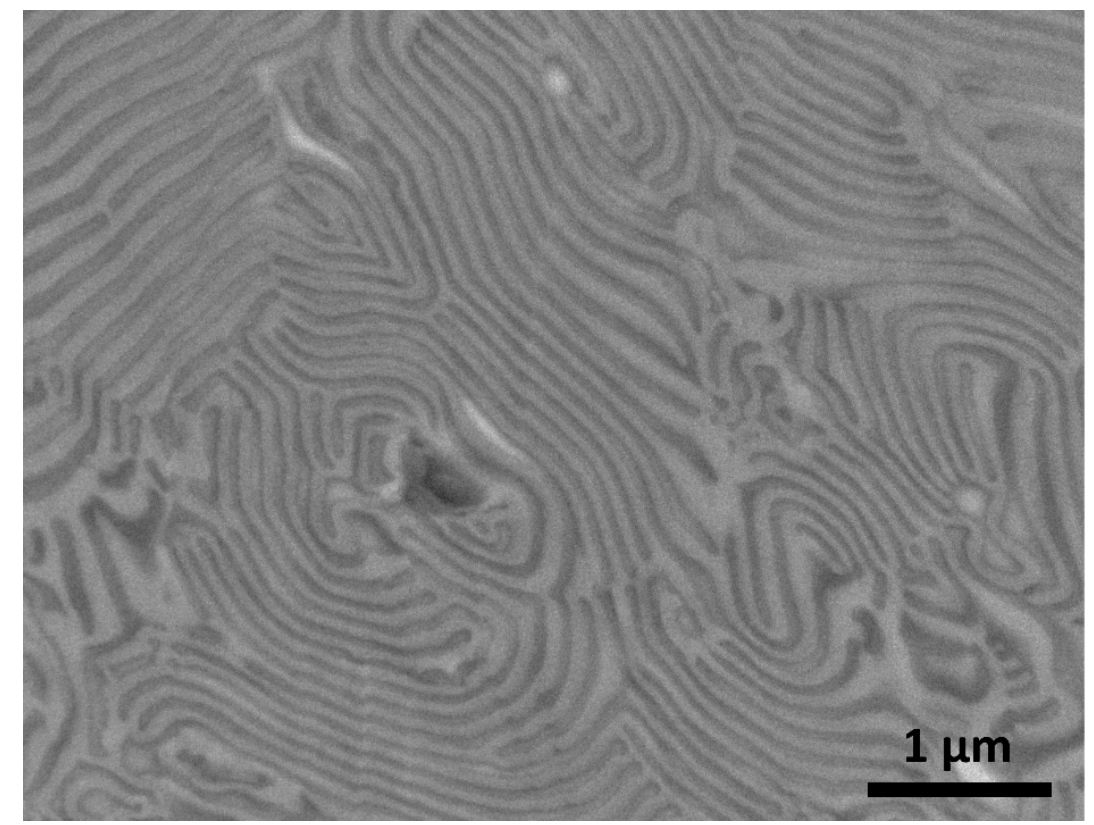

Figure S4. Cross-sectional TEM image of the chaos structure located at central part of solid PC microspheres formed by PS213k-b-P2VP215k. 


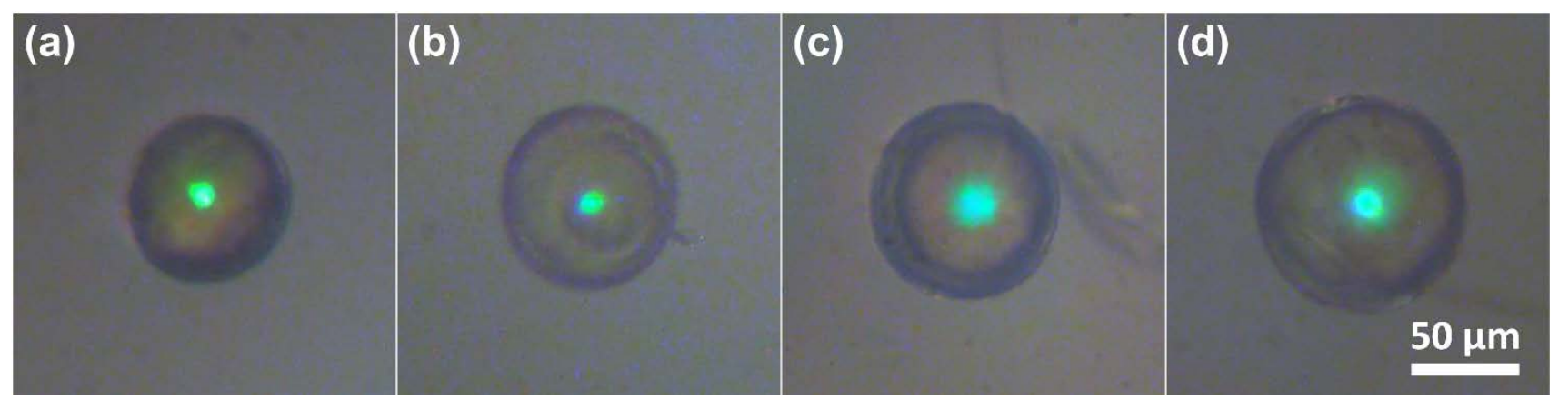

Figure S5. OM images of PC microcapsules of $\mathrm{PS}_{250 \mathrm{k}}-b-\mathrm{P} 2 \mathrm{VP}_{200 \mathrm{k}}$ obtained by changing AOT concentration in chloroform: (a) $0 \mathrm{mg} / \mathrm{mL}$, (b) $25 \mathrm{mg} / \mathrm{mL}$, (c) $30 \mathrm{mg} / \mathrm{mL}$, (d) $40 \mathrm{mg} / \mathrm{mL}$. In those cases, concentration of polymer was all $5 \mathrm{mg} / \mathrm{mL}$ and mean size of initial emulsion droplets were about $300 \mu \mathrm{m}$.

When the size of initial droplet was kept unchanged, concentration of AOT determined the volume of water droplet that eventually remained in the droplet. Therefore, higher concentration of AOT could resulted in larger cavity and overall size of PC microcapsules. In Figure S5, the cavity diameters were $0 \mu \mathrm{m}, 43 \mu \mathrm{m}, 66 \mu \mathrm{m}$, and $78 \mu \mathrm{m}$, when the concentrations of AOT were $0 \mathrm{mg} / \mathrm{mL}, 25$ $\mathrm{mg} / \mathrm{mL}, 30 \mathrm{mg} / \mathrm{mL}$, and $40 \mathrm{mg} / \mathrm{mL}$. 

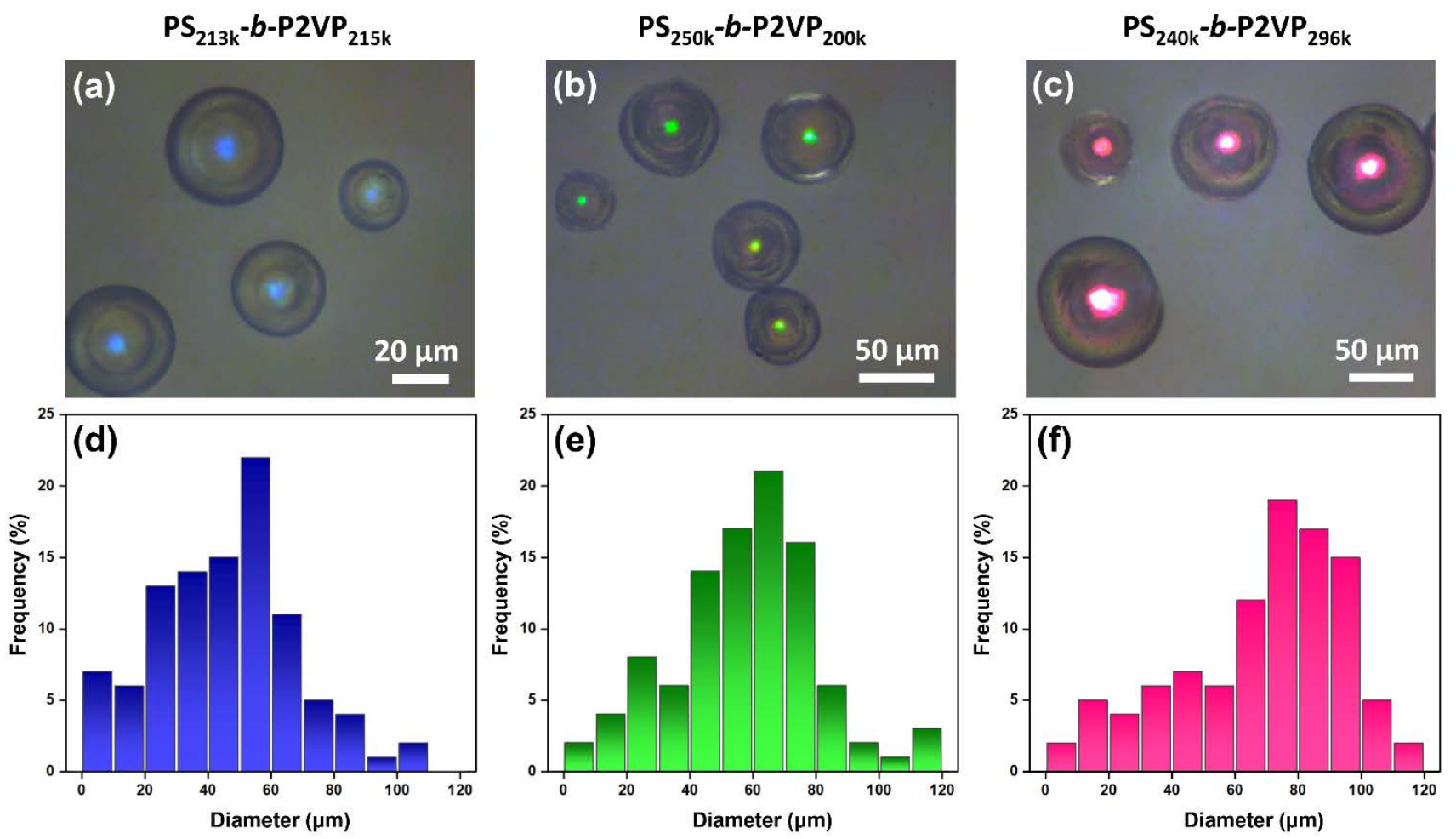

Figure S6. OM images of the PC microcapsules prepared by (a) $\mathrm{PS}_{213 \mathrm{k}-} b-\mathrm{P} 2 \mathrm{VP}_{215 \mathrm{k}}$, (b) $\mathrm{PS}_{250 \mathrm{k}-b}-\mathrm{P}_{2 \mathrm{VP}} 200 \mathrm{k}$, (c) $\mathrm{PS}_{240 \mathrm{k}}-b-\mathrm{P} 2 \mathrm{VP}_{296 \mathrm{k}}$ with polydispersed size. (d-f) are the corresponding size distribution histogram of 100 microcapsules.

The overall size of microcapsules could be varied by changing the size of initial emulsion droplets. These results indicated that structural color was mainly determined by the $M_{\mathrm{n}}$ of PS- $b$-P2VP, while little impacted by the overall size. 

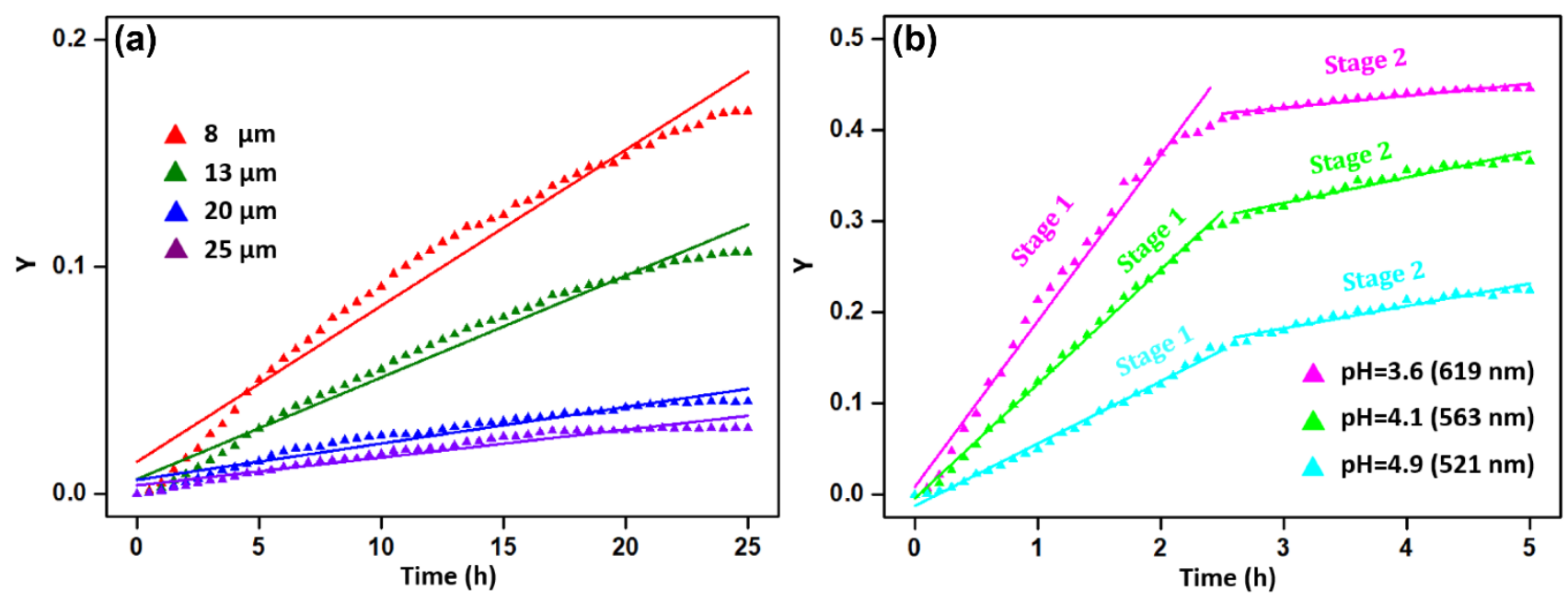

Figure S7. The linear regression analysis of the R6G release profiles of (a) microcapsules with various shell thickness in neutral water and (b) microcapsules with $8 \mu \mathrm{m}$ shell thickness at various pH values according to the Baker-Lonsdale model (Eq. S1).

The Baker-Lonsdale model (Eq. S1) is a classic model to describe the controlled release of drug from a spherical matrix, ${ }^{1}$ which can be applied to model the release behavior of the capsules. ${ }^{2,3}$

$\frac{3}{2}\left[1-\left(1-\frac{M_{t}}{M_{\infty}}\right)^{2 / 3}\right]-\frac{M_{t}}{M_{\infty}}=\frac{3 D_{m} C_{m s}}{r_{0}^{2} C_{0}} t$

In Eq. S1, $M_{\mathrm{t}}$ and $M_{\infty}$ are the drug released amount at time $t$ and an infinite time, $C_{m s}$ and $C_{0}$ are the solubility and the initial concentration of drug in the matrix, $D_{m}$ is the diffusion coefficient and ro is the radius of the spherical matrix. The equation is simplified as $Y=k t$, where $Y$ stands for $\frac{3}{2}[1-$ $\left.\left(1-\frac{M_{t}}{M_{\infty}}\right)^{2 / 3}\right]-\frac{M_{t}}{M_{\infty}}$ and the slope $k$ represents $\frac{3 D_{m} C_{m s}}{r_{0}^{2} C_{0}}$. We found that the R6G release in neutral water could be well fitted by the Baker-Lonsdale model (Figure S7a, the coefficient of determination $R^{2}>0.94$ ), indicating the release of R6G from the microcapsules with different shell thickness was diffusion-controlled. The releasing behaviors of the microcapsules at $\mathrm{pH}=4.9,4.1,3.6$ were more complicated. Each release profile could be divided into two stages, which could also be well fitted by the Baker-Lonsdale model (Figure S7b, $R^{2}>0.93$ ). 
Table S1. Releasing rate constant $(k)$ and coefficient of determination $\left(R^{2}\right)$ for R6G release from the photonic microcapsules.

\begin{tabular}{ccccccccc}
\hline & & $25 \mu \mathrm{m}$ & $20 \mu \mathrm{m}$ & $13 \mu \mathrm{m}$ & $8 \mu \mathrm{m}$ & $\mathrm{pH}=4.9$ & $\mathrm{pH}=4.1$ & $\mathrm{pH}=3.6$ \\
\hline Stage & $k / 10^{-3}$ & 1.22 & 1.60 & 4.48 & 6.86 & 68.3 & 125.6 & 182.2 \\
1 & $R^{2}$ & 0.944 & 0.940 & 0.978 & 0.971 & 0.992 & 0.997 & 0.983 \\
Stage & $k / 10^{-3}$ & & & & & 24.5 & 28.3 & 13.1 \\
2 & $R^{2}$ & & & & 0.954 & 0.952 & 0.936 \\
\hline
\end{tabular}




\section{References:}

[1] Costa, P.; Lobo, J. M. S. Modeling and Comparison of Dissolution Profiles. Eur. J. Pharm. Sci. 2001, 13, 123-133.

[2] Xu, J.; Li, J.; Yang, Y.; Wang, K.; Xu, N.; Li, J.; Liang, R.; Shen, L.; Xie, X.; Tao, J.; Zhu, J. Block Copolymer Capsules with Structure-Dependent Release Behavior. Angew. Chem., Int. Ed. 2015, 55, 14633-14673.

[3] Radin, S.; Chen, T.; Ducheyne, P. The Controlled Release of Drugs from Emulsified, Sol Gel Processed Silica Microspheres. Biomaterials 2009, 30, 850-858. 